\title{
Design und Analyse elektrisch kleiner Antennen für den Einsatz in UHF RFID Transpondern
}

\author{
R. Herschmann ${ }^{1}$, M. Camp ${ }^{2}$, and H. Eul ${ }^{1}$ \\ ${ }^{1}$ Universität Hannover, Institut für Hochfrequenztechnik und Funksysteme, Germany \\ ${ }^{2}$ Universität Hannover, Institut für Grundlagen der Elektrotechnik und Messtechnik, Germany
}

\begin{abstract}
Zusammenfassung. RFID Systeme werden seit Anfang der neunziger Jahre mit stetig zunehmender Verbreitung im Bereich der automatischen Produktidentifikation, der Diebstahlsicherung (EAS, Electronic Article Surveillance) und für automatische Zutrittskontrollsysteme eingesetzt. Objekte werden hierzu mit einem Transponder ausgestattet, der aus einer Antenne und einem Chip auf einem Trägermaterial besteht. Von großem Interesse ist die Entwicklung und Optimierung von passiven Transpondern für den Einsatz in UHF RFID Systemen. Diese Transponder beziehen die Energie zum Betrieb des Chips aus dem elektromagnetischen Feld einer Schreib/Leseeinheit. Hierfür ist neben der Anpassung der Eingangsimpedanz der Antenne an die Chipimpedanz auch eine möglichst hohe Bandbreite der Antennen wünschenswert, um die Funktion des Transponders bei Schwankungen der Chipimpedanz und variablen Umgebungsparametern zu gewährleisten. Der aus Platzgründen notwendige Einsatz elektrisch kleiner Antennen bedingt eine möglichst optimale Ausnutzung der zur Verfügung stehenden Fläche auf dem Trägermaterial zur Aufnahme der Antenne. Die vorliegende Arbeit beschreibt ein Verfahren zur Analyse und Synthese neuartiger Antennendesigns auf der Basis parametrisierter meandrierter, spiralförmiger und logarithmisch periodischer Dipole.
\end{abstract}

\section{Einleitung}

In vielen Bereichen industrieller Produktionsketten, im Bereich der Warenwirtschaft und des Handels, sowie in der Beschaffungs- und Distributionslogistik haben Systeme zur automatischen Warenerkennung und Produktverfolgung (Auto-ID) eine weite Verbreitung gefunden. Eine der bekanntesten Auto-ID Technologien beruht auf der Verwen-

Correspondence to: R. Herschmann

(herschmann@hft.uni-hannover.de) dung von Barcode-Etiketten. Zunächst haben sich zu Beginn der neunziger Jahre zusätzlich RFID Systeme (Radio Frequency Identification) basierend auf induktiv gekoppelten Funksystemen etabliert, die bis heute eine weite Verbreitung in verschiedenen Anwendungsszenarien gefunden haben. Eines der zur Zeit am stärksten wachsenden Marktsegmente im RFID Bereich besteht in der Einführung von UHF RFID Systemen. Hierbei handelt es sich um elektromagnetisch gekoppelte Funksysteme, die im Allgemeinen aus einer Schreib/Leseeinheit, Transpondern zur Warenkennzeichnung und einem IT-System zur Datenverarbeitung bestehen. Die Vorteile liegen vor allem in der größeren Reichweite sowie der fehlenden Notwendigkeit einer direkten Sichtverbindung zwischen dem Transponder und der Schreib/Leseeinheit.

Eine besondere Bedeutung in einem UHF RFID System kommt den Transponderantennen zu. Passive Transponder verfügen über keine eigene Energieversorgung. Sie müssen die zum Betrieb notwendige Energie aus dem elektromagnetischen Feld der Schreib/Leseeinheit aufnehmen. Große Reichweiten im Bereich mehrerer Meter verlangen demzufolge eine gute Anpassung der Antenne an die Chipimpedanz. Die Impedanzeigenschaften der Antennen werden durch die Materialparameter des zu kennzeichnenden Objekts stark beeinflusst. Die Einflüsse der Umgebung sowie die herstellungsbedingten und leistungsabhängigen Schwankungen der Impedanzen der Transponderchips müssen durch eine ausreichende Bandbreite der Transponderantennen berücksichtigt werden. Die üblicherweise beschränkten Abmessungen der Objekte, auf denen ein Transponder angebracht wird, führen zum Einsatz elektrisch kleiner Antennen. Basierend auf einer Definition nach Wheeler (1975) unterliegt die größte geometrische Ausdehnung $a$ dieser Antennen der Restriktion $a \leq \lambda / \pi$.

Gegenüber herkömmlichen resonanten Antennendesigns wird ein neues Konzept vorgestellt, das auf der Verwendung von elektrisch kleinen Dipolen auf der Basis von Spiralantennen, logarithmisch periodischen Designs und Me-

Published by Copernicus GmbH on behalf of the URSI Landesausschuss in der Bundesrepublik Deutschland e.V. 


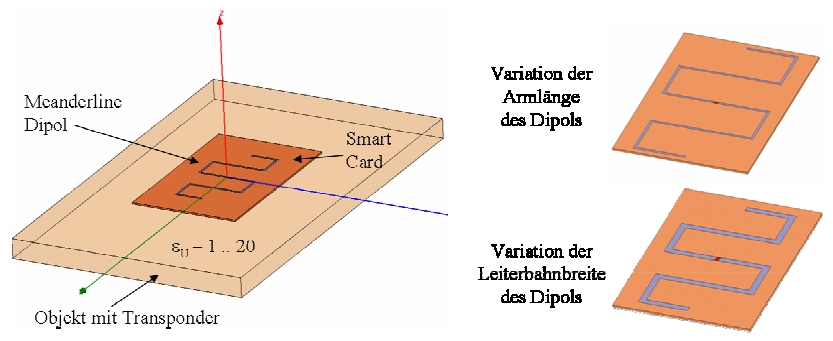

Abbildung 1. Das Simulationsmodell des parametrisierten Meanderline Dipols.

anderline Strukturen beruht. Die Analyse und Optimierung mit Hilfe eines kommerziellen Feldsimulationsprogramms erfordert die Erstellung parametrisierter Simulationsmodelle, um die Möglichkeiten der Veränderung einer geometrischen Grundstruktur zur Variation der elektromagnetischen Eigenschaften der Antenne ausschöpfen zu können. Die Extraktion relevanter Antennendesigns erfolgt in einem automatisierten Prozess durch die numerische Analyse der Impedanzcharakteristika aller simulierten Strukturkombinationen. Anhand verschiedener Designs werden die Abhängigkeiten zwischen den geometrischen Ausführungsformen der Dipolantennen und der frequenzabhängigen Eingangsimpedanz untersucht. Für die Erstellung parametrisierter Antennen und die Auswertung der Ergebnisse der numerischen Feldsimulation wird das Programm Matlab der Firma Mathworks Inc. verwendet. Alle Feldsimulationen werden mit dem 3-D-Feldsimulatiosprogramm HFSS der Firma Ansoft Inc. durchgeführt.

\section{Parametrisierte Antennen}

Die Notwendigkeit der Parametrisierung der geometrischen Strukturen ergibt sich aus der Tatsache, dass der Einfluss von Umgebungsparametern (Materialparameter des Substratträgers und des zu kennzeichnenden Objekts) auf die Eingangsimpedanz der Antenne bei variabler Geometriestruktur nicht mit Hilfe von analytischen Ausdrücken berechnet werden kann. Die Parametrisierung der Antennendesigns bietet eine effektive Möglichkeit, die Antennenimpedanz bei Resonanz an den Realteil der Chipimpedanz, der herstellerabhängig in einem Bereich von $\operatorname{Re}\left\{\underline{z}_{\mathrm{Chip}}\right\} \approx 5 \Omega-50 \Omega$ liegen kann, anzupassen. Überdies muss der Imaginärteil der Eingangsimpedanz der Antenne den meist stark kapazitiven Blindanteil der Chipimpedanz bei der gewünschten Resonanzfrequenz kompensieren. Auf die Einführung eines aus konzentrierten oder aus Leitungselementen bestehenden Anpassungsnetzwerks wird aus Kostengründen und den Restriktionen hinsichtlich des Platzangebots für die zusätzlichen Elemente verzichtet. Die Antenne übernimmt in dieser Ausführungsform die Wandlung der elektromagnetischen

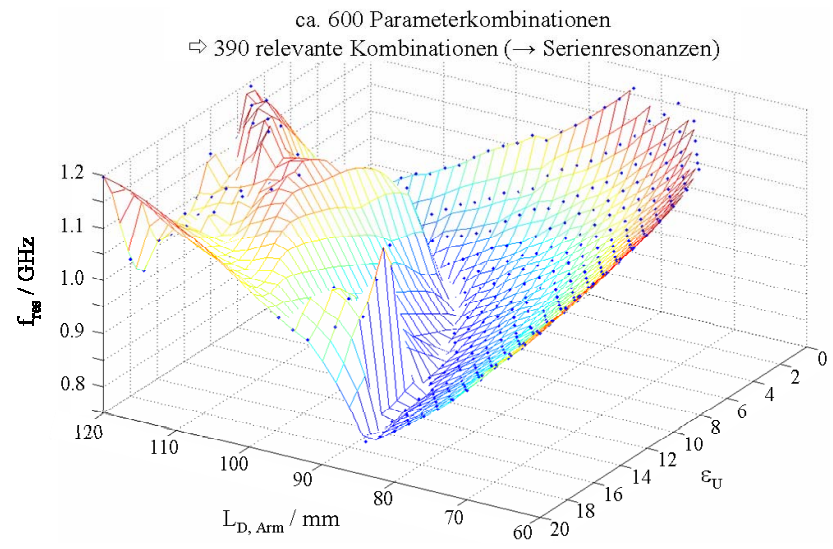

Abbildung 2. Die Resonanzfrequenz in Abhängigkeit der Armlänge des Dipols und der Permittivitätszahl des Objekts.

Freiraumwelle in eine leitungsgeführte Welle und die Impedanzanpassung an den Transponderchip.

Das Prinzip der Analyse und Synthese einer Transponderantenne auf der Basis einer parametrisierten geometrischen Struktur wird im Folgenden am Beispiel eines Meanderline Dipols erläutert. Die Berandung einer Antenne besteht aus einer Punktemenge, die mit Hilfe eines Mathematikprogramms erzeugt werden kann. Für die Parametrisierung besteht der wesentliche Schritt darin, diese Punktemenge nicht durch die explizite Angabe der numerischen Werte der zugehörigen Koordinaten sondern vielmehr die Geometrie durch die Einführung geeigneter Variablen in eine durch mathematische Berechnungsvorschriften definierte Struktur zu überführen. Die Formeln werden dabei explizit zur Beschreibung der Lage eines jeden Punktes der Antenne in der Ebene oder im Raum als Funktion definierter Parameter eingeführt. Für den in Abb. 1 dargestellten Meanderline Dipol werden die Länge und die Breite der Leiterbahn als Parameter definiert. Der Dipol ist auf einem Trägersubstrat im Smart Card Format aufgebracht. Der Tranponder ist wiederum auf einem Objekt mit variablen dielektrischen Eigenschaften befestigt. Für die Einstellung einer bestimmten Resonanzfrequenz bei festgelegter Permittivitätszahl $\varepsilon_{U}$ des Objekts muss eine optimale Kombination der Armlänge des Dipols $L_{D}$, Arm und der Leiterbahnbreite $B_{D}$ aus den Ergebnissen eines Parametersweeps der numerischen Feldanalyse extrahiert werden. Der Parametersweep wird hierzu zunächst mit einem groben Raster der beteiligten geometrischen $\mathrm{Pa}-$ rameter unter Berücksichtigung der variablen Materialparameter durchgeführt. In Abb. 2 ist das Ergebnis der Auswertung eines Parametersweeps hinsichtlich der Resonanzfrequenz dargestellt, bei dem ausschließlich die Dipollänge und die Permittivität des Objekts variiert wurden. Die Ergebnisse werden bezüglich aller relevanten Parameterkombinationen, die in einem definierten Frequenzbereich eine oder mehrere Resonanzen aufweisen, gefiltert und nach Serien- und Parallelresonanzen separiert. Diejenigen geometrischen Parame- 


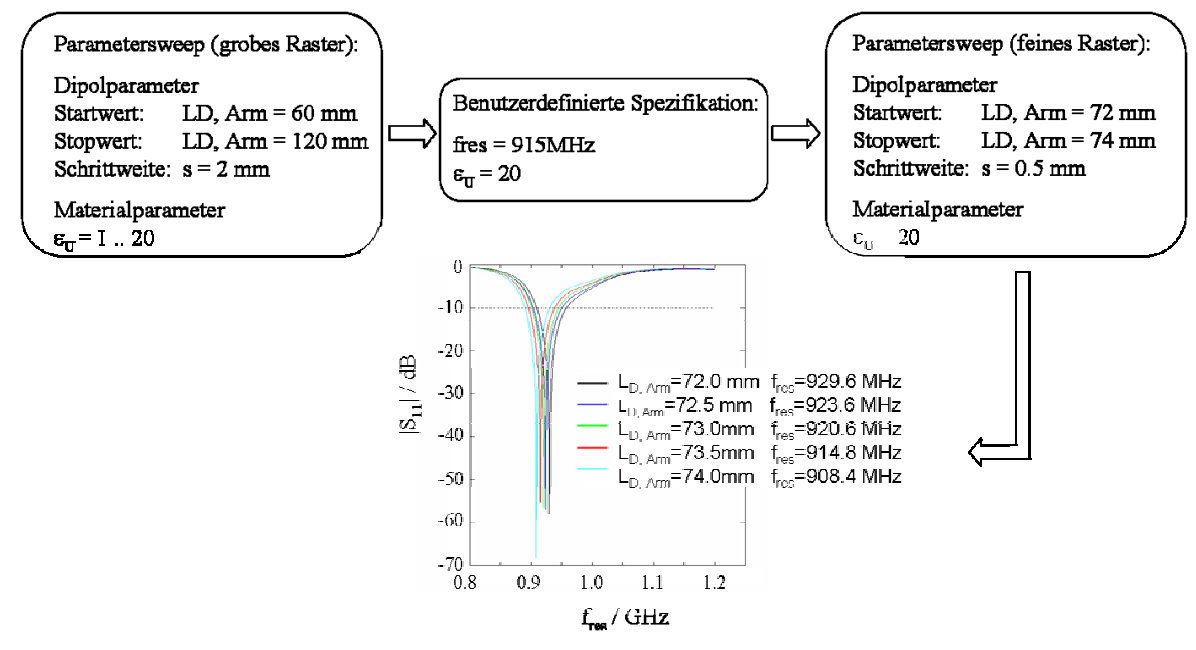

Abbildung 3. Das Prinzip der Vorgehensweise zur Extraktion optimierter Antennendesigns am Beispiel des Meanderline Dipols bei Vorgabe der Resonanzfrequenz und der Permittivitätszahl des Objekts.
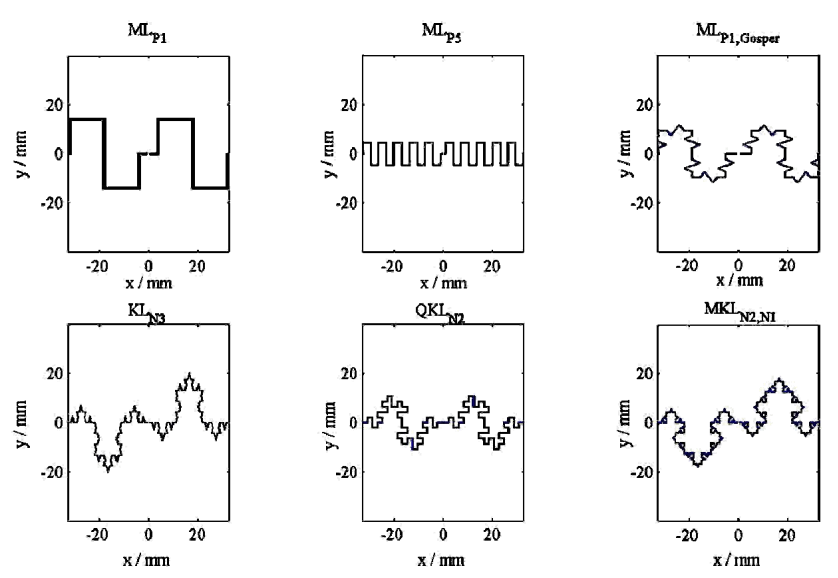

Abbildung 4. Designvarianten meandrierter Dipole mit konstanter Leiterbahnbreite.

terkombinationen, die spezifizierten elektromagnetischen Eigenschaften bezüglich der Resonanzfrequenz, der Bandbreite und dem Realteil der Antennenimpedanz am nächsten liegen, dienen unter Berücksichtigung der Vorgabewerte für die Materialparameter des Objekts als Ausgangspunkt eines weiteren Parametersweeps mit einem feineren Raster oder auch einer abschließenden Optimierung. Die notwendigen Schritte werden am Beispiel des Meanderline Dipols für die Adaption der Geometrieparameter an eine Resonanzfrequenz von $f=915 \mathrm{MHz}$ bei einem Wert der Permittivitätszahl des Objekts von $\varepsilon_{U}=20$ in Abb. 3 verdeutlicht. Das unter Verwendung von Matlab und Ansoft HFSS implementierte und automatisierte Verfahren wurde in Herschmann and Eul (2004) eingeführt und bildet die Grundlage für die im Folgenden vorgestellten Untersuchungen an Antennendesigns mit konstanter Leiterbahnbreite.

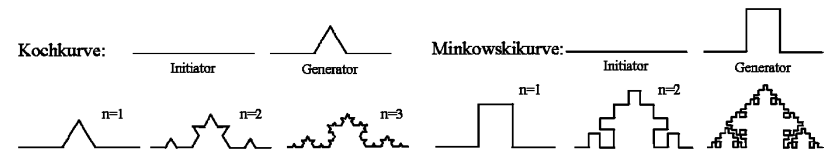

Abbildung 5. Die Erzeugung präfraktaler Kurven unter Anwendung eines iterierten Funktionensystems (IFS).

\section{Meandrierte Dipolantennen konstanter Leiterbahnbreite}

In Anlehnung an Untersuchungen an Dünndrahtstrukturen in Best (2004) werden die Eigenschaften von meandrierten planaren Dipolen mit konstanter Leiterbahnbreite diskutiert. In Abb. 4 sind die Geometrien der untersuchten Antennen dargestellt. Alle Antennen unterscheiden sich deutlich hinsichtlich ihrer strukturellen Parameter. So finden sich neben Meanderline Dipolen $\left(\mathrm{ML}_{P 1}, \mathrm{ML}_{P 5}\right)$, eine Kombination aus Meanderline und präfraktaler Struktur ( $\mathrm{ML}_{P 1, \mathrm{Gosper}}$ ), präfraktale $\left(\mathrm{KL}_{N 3}, \mathrm{QKL}_{N 2}\right)$ und kombinierte präfraktale Strukturen $\left(\mathrm{MKL}_{N 2, N 1}\right)$. Präfraktale Kurven werden durch ein iteriertes Funktionensystem (IFS) erzeugt. Ein IFS generiert auf der Basis eines Initiators unter Anwendung eines Generators in einem iterativen Prozess aus einer geometrisch einfachen eine geometrisch komplexere Struktur. Die Eigenschaften des Generators werden durch affinlineare Transformationen beschrieben. In Abb. 5 ist der Erzeugmechanismus anhand bekannter Beispiele von fraktalen Kurven verdeutlicht. Die Darstellungen der Koch- und Minkowskikurve sind bis zu einer Iterationstiefe $n=3$ skizziert. Die affinlinearen Transformationen des IFS können dabei die Operationen Skalierung, Drehung, Scherung und Spiegelung umfassen. Eine fraktale Struktur entsteht durch die Anwendung eines iterierten Funktionensystems, wenn der Iterationsprozess für $n \rightarrow \infty$ durchgeführt wird. Derartige geometrische Strukturen sind technisch nicht reproduzierbar und somit ir- 
Tabelle 1. Geometrische und elektrische Eigenschaften meandrierter Strukturvarianten im Bereich der ersten Serienresonanz.

\begin{tabular}{|c|c|c|c|c|c|c|}
\hline \multirow{3}{*}{$\begin{array}{l}\text { Antennen- } \\
\text { design }\end{array}$} & \multicolumn{2}{|c|}{ Max. Ausdehnung in } & \multirow{3}{*}{$\begin{array}{l}\text { Armlänge } \\
\frac{L_{D, \text { Arm }}}{\mathrm{mm}}\end{array}$} & \multirow{3}{*}{$\begin{array}{l}\text { Resonanz- } \\
\text { frequenz } \\
\frac{f_{\text {res }}}{\mathrm{GHz}}\end{array}$} & \multirow{3}{*}{$\begin{array}{l}\text { Strahlungs- } \\
\text { widerstand } \\
\qquad \frac{R_{S}}{\Omega}\end{array}$} & \multirow{3}{*}{$\begin{array}{c}\text { Güte } \\
Q\end{array}$} \\
\hline & $\mathrm{x}$-Richtung & y-Richtung & & & & \\
\hline & $\frac{l_{x}}{\mathrm{~mm}}$ & $\frac{l_{y}}{\mathrm{~mm}}$ & & & & \\
\hline $\mathrm{ML}_{P 1}$ & 65.4 & 28.8 & 87.87 & 1.010 & 16.68 & 174 \\
\hline $\mathrm{ML}_{P 5}$ & 65.4 & 9.1 & 120.67 & 0.995 & 16.03 & 213 \\
\hline $\operatorname{ML}_{P 1, \text { Gosper }}$ & 65.4 & 23.8 & 110.10 & 1.010 & 16.59 & 165 \\
\hline $\mathrm{KL}_{N 3}$ & 65.4 & 40.2 & 121.57 & 0.995 & 15.12 & 198 \\
\hline $\mathrm{QKL}_{N 2}$ & 65.4 & 21.6 & 132.96 & 0.996 & 14.58 & 207 \\
\hline $\mathrm{MKL}_{N 2, N 1}$ & 65.4 & 36.3 & 129.15 & 0.996 & 15.36 & 193 \\
\hline
\end{tabular}
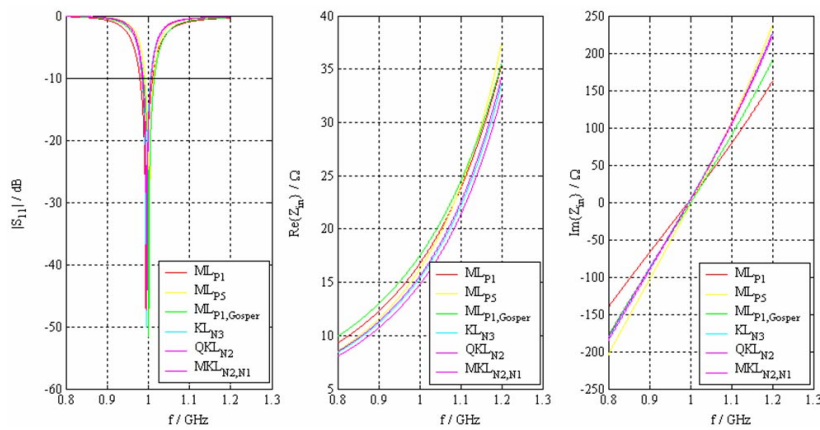

Abbildung 6. Die Rückflussdämpfung und Eingangsimpedanz meandrierter Antennen als Funktion der Frequenz im Bereich der ersten Serienresonanz.

relevant. Wird der Iterationsprozess hingegen nach einer endlichen Anzahl von Schritten abgebrochen, so entsteht eine präfraktale Geometrie.

Die in Abb. 4 dargestellten Strukturen sind punktsymmetrisch zum Ursprung des Koordinatensystems und weisen die gleiche maximale Ausdehnung entlang ihrer Hauptachse auf. Die Antennen sind durch Anpassung der geometrischen Parameter auf eine Resonanzfrequenz von $f_{\text {res }}=1 \mathrm{GHz}$ abgestimmt. In Tabelle 1 sind neben den geometrischen Daten auch die Impedanz und die Güte der Antennen aus der numerischen Feldanalyse für den Bereich der Resonanzfrequenz zusammengefasst. Die Ergebnisse verdeutlichen eine erhebliche Restriktion bezüglich des Ansatzes, durch Variation der Geometrie eine Anpassung der Impedanz des Transponderchips an die Eingangsimpedanz der Antenne zu ermöglichen. Dieses Phänomen wurde bereits in Best (2004) basierend auf einer ausführlichen Studie an Dünndraht Monopolantennen in geometrisch unterschiedlichen Variationen erörtert. Der für alle Designvarianten nahezu konstante Wert des Realteils der Antennenimpedanz bei Resonanz ist direkt mit der geo- metrischen Ersatzgröße der effektiven Länge gemäß Gl. (1) verknüpft.

$l_{\mathrm{eff}}=2 \cdot \sqrt{A_{W} \cdot \frac{R_{S}}{\eta_{0}}}=\frac{\lambda}{\pi} \cdot \sqrt{G \cdot \frac{R_{S}}{120 \Omega}}$

Da die Antennen verlustfrei simuliert wurden, entspricht der Realteil der Antennenimpedanz dem Strahlungswiderstand $R_{S}$. Der Antennengewinn liegt für alle Antennen aus Abb. 4 in einem Bereich von $G \approx 1.6$. Die Abweichungen der Gütewerte in Tabelle 1 resultieren hauptsächlich aus dem unterschiedlichen Verlauf des frequenzabhängigen Imaginärteils der Antennen in Abb. 6. Nach Wheeler (1975) ist das effektive Volumen der Antennen über das Volumen einer Kugel oder über das äquivalente Volumen eines Quaders definiert. Der Zusammenhang zwischen dem Radius $r_{V_{\text {eff }}}$ der das effektive Volumen repräsentierenden Kugel, der Antennengüte und der Wellenlänge lautet

$r_{V_{\text {eff }}}=\frac{\lambda}{\pi} \cdot\left(\frac{9}{2 \cdot Q}\right)^{\frac{1}{3}}$

Je höher die Güte ist, desto kleiner ist das effektive Volumen der Antennen. Demzufolge weist ein nur mäßig gefalteter Dipol, welcher die quer zur Längsachse der Antenne zur Verfügung stehende Fläche besser ausnutzt als ein vergleichbar stark gefalteter Dipol, ein größeres effektives Volumen auf. Ein kleinerer Wert des effektiven Volumens führt bei einem stark gefalteten Dipol zu einem Verlauf des Imaginärteils mit höherer Steigung im Bereich der ersten Serienresonanz als bei einem vergleichbaren Dipol mit weniger stark ausgeprägter Faltung. Die Auswirkung des effektiven Volumens auf die Performance der Antenne zeigt sich besonders deutlich im Vergleich der meandrierten Dipolstrukturen $\mathrm{ML}_{P 1}$ und $\mathrm{ML}_{P 5}$. Je höher der Grad der Faltung einer Struktur bei gleicher Resonanzfrequenz und gleicher maximaler Ausdehnung in Hauptachsenrichtung der Antenne ist, 

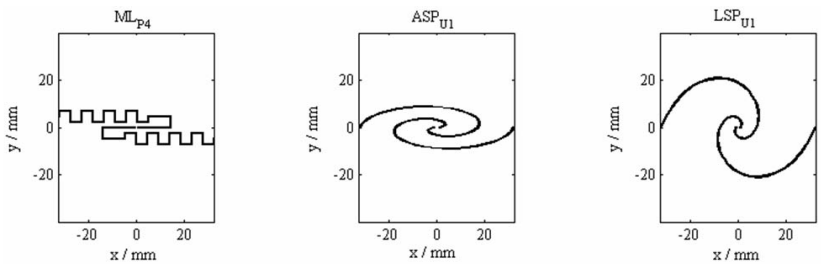

$\mathrm{LPR}_{\mathrm{PB}}$
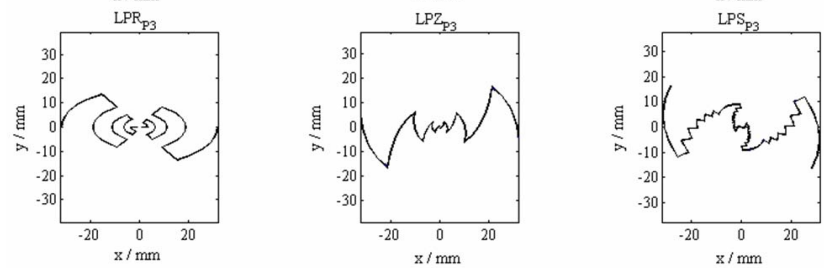

Abbildung 7. Designvarianten logarithmisch periodischer und spiralförmiger Dipolantennen mit konstanter Leiterbahnbreite.

desto größer muss die Gesamtlänge werden, um eine Einhaltung der Resonanzfrequenz zu gewährleisten. Der notwendige Zuwachs an Leitungslänge ist hierbei von den Verkopplungseigenschaften der Strukturelemente der Dipolarme abhängig. Während eine Erhöhung der Leiterlänge einen Anstieg der Induktivität zur Folge hat, führt die Faltung der Struktur zum Auftreten parasitärer Verkoppelungen zwischen benachbarten Strukturelementen. Die parasitären Effekte wirken kontraproduktiv zum Induktivitätsgewinn durch Leiterverlängerung und führen unweigerlich zu einer Zunahme der gespeicherten Energie und somit zu einer Erhöhung der Güte und Verringerung der Bandbreite. Die in Abb. 6 zusammengefassten Abhängigkeiten der frequenzabhängigen Verläufe von Rückflussdämpfung sowie Real- und Imaginärteil der Eingangsimpedanz der Antennen der zugrunde liegenden Geometrien aus Abb. 4 verdeutlichen diese $\mathrm{Zu}$ sammenhänge.

\section{Spiralförmige und logarithmisch periodische Dipole konstanter Leiterbahnbreite}

Die aufgezeigte Abhängigkeit zwischen einer konstanten Ausdehnung der elektrisch kleinen Dipolantennen in die Richtung der Hauptachse und dem nahezu geometrieunabhängigen Wert des Realteils der Eingangsimpedanz bei Resonanz kann durch diverse Strukturvariationen aufgehoben werden. Die in Abb. 7 vorgestellten Strukturen basieren bis auf den Dipol mit der Bezeichnung $\mathrm{ML}_{P 4}$ auf der Einführung logarithmisch periodischer Antennen sowie auf der Verwendung von Dipolen auf der Basis von Spiralen. Auch diese Antennen sind durch Anpassung der geometrischen Parameter auf eine Resonanzfrequenz von $f_{\text {res }}=1 \mathrm{GHz}$ abgestimmt. Diese Geometrien werden eingesetzt, da aufgrund des Erzeugungsmechanismus Strukturen entstehen, die aufgrund der damit verbundenen Transformation vom Original- in den Bildbereich geometrisch gefaltet werden. Ebenso wie bei der Verwendung präfraktaler Geo-
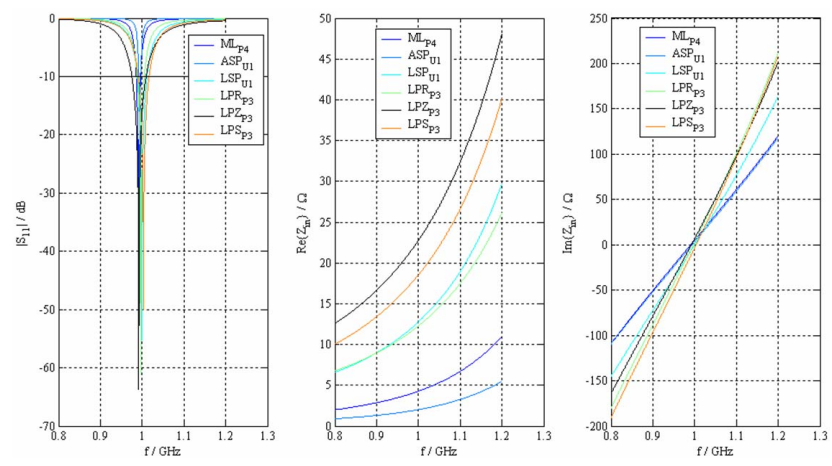

Abbildung 8. Die Rückflussdämpfung und Eingangsimpedanz spiralförmiger und logarithmisch periodischer Antennen als Funktion der Frequenz im Bereich der ersten Serienresonanz

metrien besteht somit eine Möglichkeit zur Miniaturisierung von Antennen. Entgegen dem in der Literatur häufig beschriebenen Anwendungsgebiet als Breitbandantennen werden die logarithmisch periodischen und spiralförmigen Antennen als resonante Schmalbandantennen betrieben. Die logarithmisch periodische Antenne und die winkelkonstante Spiralantenne werden durch die Anwendung der folgenden Transformationsvorschrift auf eine geometrische Struktur im Originalbereich im Bildbereich erzeugt. Der Originalbereich verfügt über die Koordinaten $(\xi, \eta)$, die durch die Transformation $f(\xi, \eta) \rightarrow w(x(\xi, \eta), y(\xi, \eta))$ auf die Koordinaten $(x(\xi, \eta), y(\xi, \eta))$ des Bildbereichs abgebildet werden. Zwischen den Koordinatensystemen des Original- und Bildbereichs besteht der Zusammenhang

$\left\{\begin{array}{l}x \\ y\end{array}\right\}=e^{\xi} \cdot\left\{\begin{array}{c}\cos (\eta) \\ \sin (\eta)\end{array}\right\}$.

Diese Antennen weisen bei gleicher geometrischer Ausdehnung in xRichtung und gleicher Resonanzfrequenz abhängig von der geometrischen Ausführung eine Variation des Realteils der Eingangsimpedanz der Antenne über eine weiten Bereich auf. Ebenso variiert die Frequenzabhängigkeit des Real- und Imaginärteils der Eingangsimpedanz der Antenne und somit die Güte und Bandbreite der Dipole. Die starken Schwankungen bedingen gegenüber den in Abb. 4 vorgestellten Strukturvarianten auch einen deutlich größeren Wertebereich der auftretenden Bandbreiten. Die Struktur mit dem kleinsten Wert der Eingangsimpedanz bei Resonanz von $\operatorname{Re}\left\{Z_{i n}\right\}=2 \Omega$, die archimedische Spirale $\operatorname{ASP}_{U 1}$, weist lediglich eine Bandbreite von $B_{-10 \mathrm{~dB}}=5 \mathrm{MHz}$ auf. Demgegenüber wird der höchste Wert der Bandbreite von $B_{-10 \mathrm{~dB}}=34 \mathrm{MHz}$ für die logarithmisch periodische Zickzackantenne $\mathrm{LPZ}_{P 3}$ bei einem Realteil der Eingangsimpedanz von $\operatorname{Re}\left\{Z_{i n}\right\}=22 \Omega$ erreicht. Der Dipol $\mathrm{ML}_{P 4}$ zeichnet sich gegenüber den in Abb. 4 dargestellten Meanderline Dipolen im Wesentlichen durch einen veränderten Speisebereich aus. Diese geometrische Variation bedingt die veränderten elektromagnetischen Eigenschaften der Anten- 
ne im Vergleich zu den in Tabelle 1 zusammengefassten Ergebnissen. Bei einer Armlänge von $L_{D, \text { Arm }}=102.3 \mathrm{~mm}$ beträgt der Strahlungswiderstand $R_{S}=4.1 \Omega$ und die Güte $Q=174$. Die Bandbreite ist gegenüber den vergleichbaren Werten der Meanderline Dipole aus Abb. 4, die im Bereich $B_{-10 \mathrm{~dB}}=(20-30) \mathrm{MHz}$ liegen, auf einen Wert von $B_{-10 \mathrm{~dB}}=10 \mathrm{MHz}$ reduziert.

\section{Zusammenfassung}

Die Untersuchung verschiedener geometrischer Strukturen zum Einsatz als Antenne in passiven RFID Transpondern hat gezeigt, das auf der Grundlage parametrisierter Antennen eine effektive Möglichkeit zur Extraktion relevanter Designvarianten bei vorgegebenen Werten für die Chipimpedanz und Materialparametern der zu kennzeichnenden Objekte besteht. Am Beispiel meandrierter Dipole wurde verdeutlicht, dass diese Klasse elektrisch kleiner Antennen trotz deutlicher struktureller Unterschiede eine nahezu gleiche Performance hinsichtlich der elektromagnetischen Eigenschaften im Resonanzbereich aufweisen kann. Die Einführung einer anderen Klasse elektrisch kleiner Antennen, nämlich resonanter logarithmisch periodischer und spiralförmiger Antennendesigns, ermöglicht hingegen die notwendige Varianz der charakteristischen Kenngrößen der Antenne zur Anpassung an Transponderchips mit herstellerabhängigen Impedanzen. Das vorgestellte Verfahren zur Analyse und Synthese von Transponderantennen für UHF RFID Systeme ist ohne Weiteres auf flächige Antennenstrukturen übertragbar, die sich gegenüber den Antennen mit konstanter Leiterbahnbreite durch eine vergrößerte Bandbreite auszeichnen.

\section{Literatur}

Wheeler, H. A.: Small Antennas, IEEE Transactions on Antennas and Propagation, 23, 462-469, 1975.

Herschmann, R. and Eul, H.: RFID Systeme für den Einsatz in UHF und Mikrowellenbereich, presented at RadioTecC, Radio Techniques and Technologies for Commerc. Commun. and Sensing Appl. Berlin, 2004.

Best, S. R.: A Discussion on the Properties of Electrically Small Self Resonant Wire Antennas, IEEE Antennas and Propagation Magazine, 46, 9-22, 2004. 